\title{
Comparative assessment of DNA methylation patterns between reduced representation bisulfite sequencing and Sequenom EpiTyper methylation analysis
}

\begin{abstract}
Aim: Validation of sequencing-based DNA methylation data is an important step for meaningful translation of findings. However, there has been limited assessment of different platforms to validate methylation data from next generation sequencing. Methods: We performed a comparative methylation analysis between the genomewide platform of reduced representation bisulfite sequencing with a targeted, Sequenom EpiTyper platform (four genes were analyzed in 15 cell lines covering 52 CpG sites). Results: We show that the accuracy of validation substantially improves if results from multiple and adjacent $\mathrm{CpG}$ sites are combined rather than at single CpG sites. We demonstrate increased read number improves accuracy of reduced representation bisulfite sequencing results. Further, by using series of replicates, we document variation in samples analyzed by Sequenom EpiTyper, indicating the importance of including replicates to increase precision. Conclusion: The results reveal potential sources of bias and provide a guideline for refining study design for DNA methylation analysis.
\end{abstract}

First draft submitted: 13 December 2016; Accepted for publication: 21 February 2017; Published online: 30 May 2017

Keywords: CpG site $\bullet$ DNA methylation $\bullet$ read number $\bullet$ replicate $\bullet$ RRBS $\bullet$ Sequenom EpiTyper • variation

The advent of next generation sequencing (NGS) technologies and the progressive increase in sequence throughput has allowed the quantification of DNA methylation on an unprecedented scale. Reduced representation bisulfite sequencing (RRBS) is a frequently used NGS-based method that allows genome-wide profiling of the DNA methylome. By enriching for $\mathrm{CpG}$ sites, RRBS covers $13.4 \%$ of $\mathrm{CpG}$ sites and includes information from $80 \%$ of $\mathrm{CpG}$ islands in the human genome [1], thereby providing a cost effective alternative to whole genome bisulfite sequencing.

NGS-based techniques, such as RRBS, are commonly used as a discovery platform to generate a list of regions showing significant methylation differences between experimental groups. The next essential step is to validate these results by using a different technique either in the same samples (technical validation) or in independent cohorts (biological validation) [2]. There are several aspects in genome-wide studies that could lead to false identification of differential methylation, such as inadequate sequencing depth, the use of inappropriate statistical tests, overestimation of statistical significance from multiple testing and technical biases (e.g., PCR amplification bias). Thus an important question that arises in any large-scale DNA methylation analysis is whether the measured methylation values are accurate. Therefore, validation of results from genome-wide methylation profiles is of high importance for meaningful translation of the findings.

Several techniques allow locus-specific interrogation of methylation [3] including
Aniruddha Chatterjee ${ }^{*, \neq 1,1,2}$, Erin C Macaulay ${ }^{\ddagger 1}$, Antonio Ahn', Jackie L Ludgate', Peter A Stockwell ${ }^{3}$, Rob J Weeks ${ }^{1}$, Matthew F Parry ${ }^{4}$, Timothy J Foster ${ }^{1}$, Ingrid M Knarston', Michael R Eccles ${ }^{1,2}$ \& Ian M Morison ${ }^{1}$

'Department of Pathology, Dunedin School of Medicine, University of Otago, 270 Great King Street, Dunedin 9054, New Zealand

${ }^{2}$ Maurice Wilkins Centre for Molecular Biodiscovery, Level 2, 3A Symonds Street, Auckland, New Zealand

${ }^{3}$ Department of Biochemistry, University of Otago, 710 Cumberland Street, Dunedin 9054, New Zealand ${ }^{4}$ Department of Mathematics \& Statistics, University of Otago, PO Box 56, Dunedin, 9054, New Zealand

*Author for correspondence: Tel.: +6434703455 aniruddha.chatterjee@otago.ac.nz ${ }^{\ddagger}$ Authors contributed equally 
the Sequenom EpiTyper DNA methylation assay. Sequenom EpiTyper is a highly quantitative assay that combines base-specific enzymatic cleavage and MALDI-TOF (matrix-assisted laser desorption/ionization-time of flight) mass spectrometry to quantify individual CpG-site methylation [4]. Sequenom EpiTyper is commonly used as a validation platform for DNA methylation analyses due to its reputation of high precision [5-9].

In this study, we analyzed four genes from 15 RRBS libraries generated from human cell lines. We assessed the agreement between single CPG sites and larger regions in these genes (containing multiple $\mathrm{CpG}$ sites). We also assessed the level of variation at different read depth in RRBS data. Further, by performing several replicate experiments, we demonstrate technical variation in Sequenom EpiTyper methylation assays.

\section{Results}

Generating genome-wide \& Sequenom EpiTyper methylation profiles for 15 cell lines

Genome-scale methylation profiles were obtained from 15 human cell lines by using RRBS. Four genes (CBX8, HES5, POU3F2, EXOC3L2, see Tables 1 \& 2) were selected for comparison with the Sequenom EpiTyper assay. These regions were selected from an independent RRBS analysis [10] due to their good sequencing coverage (median read depth per $\mathrm{CpG}$ site was 38). In addition, these regions represented a range of methylation value (from 0 to $100 \%$ methylation) in the analyzed samples and therefore provide the opportunity for a comparative analysis at different methylation values. The analyzed Sequenom EpiTyper amplicon lengths varied from 62 to $454 \mathrm{bp}$. In total, $52 \mathrm{CpG}$ sites were assessed by both techniques (i.e., RRBS and Sequenom EpiTyper) and the comparative data described in this article were based on these sites (Supplementary Table 1; the primer sequences are given in Supplementary Table 2).

\section{Agreement between RRBS \& Sequenom EpiTyper assay increases when a contig of CpG sites is analyzed}

First, we compared the methylation status of each commonly covered $\mathrm{CpG}$ site for the analyzed genes. In some closely adjacent $\mathrm{CpG}$ sites, the Sequenom EpiTyper platform is unable to resolve methylation values for individual sites and an aggregated methylation value is calculated (examples can be seen in the $\mathrm{x}$-axis of Figure 3). To derive equivalent data for the comparison with RRBS, we took the mean methylation values of these aggregated CpG sites. The Bland-Altman analysis indicated that the standard deviations of the difference in these four genes were $0.18,0.16,0.21$ and 0.054 for CBX8, HES5, EXOC3L2 and POU3F2, respectively (Table 1). The Pearson's correlation coefficients (r) were $0.86,0.82,0.77$ and 0.99 , respectively. Next, we determined the extent to which a fragmentbased analysis enhanced the agreement between methods compared with single $\mathrm{CpG}$ site comparison (Supplementary Figures 1-4). The standard deviations of the differences between the fragment quantification methods were $0.042,0.085,0.10$ and 0.031 , respectively. For all four genes, the correlation between measured methylation increased $(r=0.99,0.95,0.96$ and 0.998 , respectively). Combining the data from the four genes yielded a correlation of 0.89 for all the $52 \mathrm{CpG}$ sites ( $S D$ of difference $=0.16$, see Figure $1 A \& B$ ), and when the fragment methylation values were compared, the correlation was 0.98 (SD of the difference $=0.072$, Figure $1 C \& D)$.

\section{Relationship of sequenced read number \& methylation differences}

Next, we examined the size of the effect of increased read depth on agreement between these two techniques. We analyzed the difference between RRBS and Sequenom EpiTyper methylation values with incremental read depth (step size of 5; the distribution of the RRBS read depth is shown in Supplementary Figure 5) and found, as expected, that the difference decreases with increasing read depth (Figure 2). For RRBS read depths of $\geq 10$, the correlation coefficient for CpG sites with an RRBS read depth $\geq 10$ was 0.908 (95\% CI: 0.888-0.925) (see Table 2). At a read depth of $\geq 20$, the correlation coefficient was 0.918 (95\% CI: $0.898-0.934)$, whereas at a read depth of $\geq 50$, the correlation coefficient was 0.960 (95\% CI: $0.945-0.971$ ) (Table 2).

\section{Assessment of technical variation of the}

\section{Sequenom EpiTyper platform}

To assess variability of the Sequenom EpiTyper analysis process (PCR plus mass spectrometry), we used DNA from a normal melanocyte cell line (Hemn-LP). A single aliquot of DNA from the Hemn-LP cell line was bisulfite converted and used to generate the technical replicates for the subsequent experiments (described in Figure 3). We performed PCRs on replicate samples for three genes (HES5, EXOC3L2, POU3F2). In total, the length of the amplicons varied from 218 to $437 \mathrm{bp}$, and contained $81 \mathrm{CpG}$ sites from which 32 quantifiable methylation values could be obtained (i.e., analyzable in Sequenom EpiTyper platform [Supplementary Table 1]). Of the 81 Sequenom EpiTyper CpG sites, 38 were also covered by RRBS. For POU $3 F 2$ and HES5, there were seven replicate samples, whereas for EXOC3L2, there were four (Figure $3 \mathrm{~A}-\mathrm{C}$ ). Sequenom 
Table 1. Comparative validation analysis of reduced representation bisulfite sequencing versus Sequenom EpiTyper platform at single CpG sites and over an amplicon.

\begin{tabular}{|c|c|c|c|c|c|}
\hline \multirow[t]{2}{*}{ Nearest gene } & \multirow[t]{2}{*}{ Feature } & \multirow{2}{*}{$\begin{array}{l}\text { CpG correlation (Sequenom } \\
\text { EpiTyper-RRBS) } \\
\text { r-value }\end{array}$} & \multicolumn{3}{|c|}{ Bland-Altman analysis } \\
\hline & & & Difference & SD of difference & $\begin{array}{l}\text { Limits of } \\
\text { agreement }\end{array}$ \\
\hline$C B \times 8$ & Promoter & 0.859 & -0.0110 & 0.179 & $-0.362-0.340$ \\
\hline HES5 & Promoter, CGI core & 0.817 & -0.014 & 0.160 & $-0.329-0.301$ \\
\hline EXOC3L2 & Promoter & 0.770 & -0.040 & 0.209 & $-0.451-0.370$ \\
\hline POU3F2 & Promoter, CGI core & 0.992 & -0.003 & 0.054 & $-0.110-0.103$ \\
\hline \multirow[t]{2}{*}{ All CpG sites } & NA & 0.896 & -0.017 & 0.164 & $-0.338-0.304$ \\
\hline & & $\begin{array}{l}\text { Common amplicon } \\
\text { correlation (Sequenom } \\
\text { EpiTyper-RRBS) }\end{array}$ & \multicolumn{3}{|c|}{ Bland-Altman analysis } \\
\hline$C B \times 8$ & Promoter & 0.990 & -0.012 & 0.042 & $-0.096-0.071$ \\
\hline HES5 & Promoter, CGI core & 0.953 & -0.023 & 0.085 & $-0.1907-0.1436$ \\
\hline EXOC3L2 & Promoter & 0.959 & -0.042 & 0.104 & $-0.246-0.162$ \\
\hline POU3F2 & Promoter, CGI core & 0.998 & -0.004 & 0.031 & $-0.066-0.058$ \\
\hline $\begin{array}{l}\text { All common } \\
\text { amplicons }\end{array}$ & NA & 0.982 & -0.020 & 0.072 & $-0.161-0.120$ \\
\hline
\end{tabular}

EpiTyper methylation data for individual CpG sites were obtained for each of these replicates.

The methylation values of each replicate sample at individual CpG sites are shown in Figure 3D-F. We found that many of the CpG sites showed substantial variation. For example, CpG47 for EXOC3L2 $(\mathrm{SD}=0.20$, coefficient of variation $(\mathrm{CV})=109 \%)$ and $\mathrm{CpG} 11-13$ for POU3F2 $(\mathrm{SD}=0.16$ and $\mathrm{CV}=$
59\%, Figure 3 and Supplementary Data 1 contains detailed information for the individual CpG sites). We observed a general trend of higher variability (i.e., higher $\mathrm{SD}$ ) if the methylation value is intermediate in the replicates (Figure 4). When the methylation values of the CpGs in each amplicon were averaged, the replicate samples showed better agreement: the SDs of the replicates for the amplicon were 0.05, 0.04

Table 2. Analysis of read depth and methylation correlation between reduced representation bisulfite sequencing and Sequenom EpiTyper.

\begin{tabular}{|llll|}
\hline Read cut off & Pearson co-efficient & Data points, $\mathbf{n}$ & $95 \% \mathbf{C l}$ \\
\hline No cut off & 0.896 & 383 & $0.875-0.914$ \\
\hline 5 & 0.895 & 378 & $0.873-0.913$ \\
\hline 10 & 0.908 & 356 & $0.888-0.925$ \\
\hline 15 & 0.912 & 329 & $0.892-0.928$ \\
\hline 20 & 0.918 & 295 & $0.898-0.934$ \\
\hline 25 & 0.930 & 262 & $0.912-0.945$ \\
30 & 0.932 & 238 & $0.913-0.946$ \\
\hline 35 & 0.942 & 208 & $0.925-0.956$ \\
\hline 40 & 0.953 & 188 & $0.938-0.964$ \\
\hline 45 & 0.959 & 159 & $0.945-0.970$ \\
\hline 50 & 0.960 & 147 & $0.945-0.971$ \\
\hline 55 & 0.967 & 125 & $0.953-0.976$ \\
\hline 60 & 0.971 & 111 & $0.958-0.980$ \\
\hline
\end{tabular}




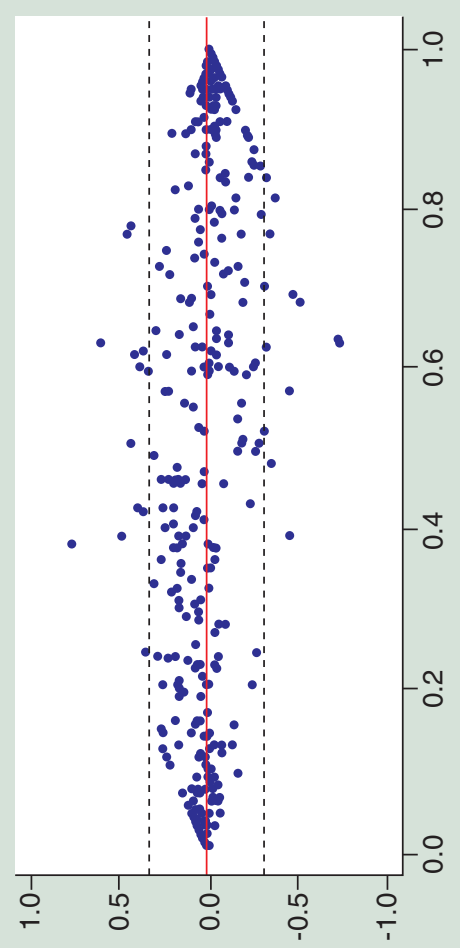

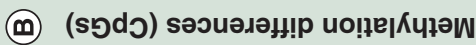

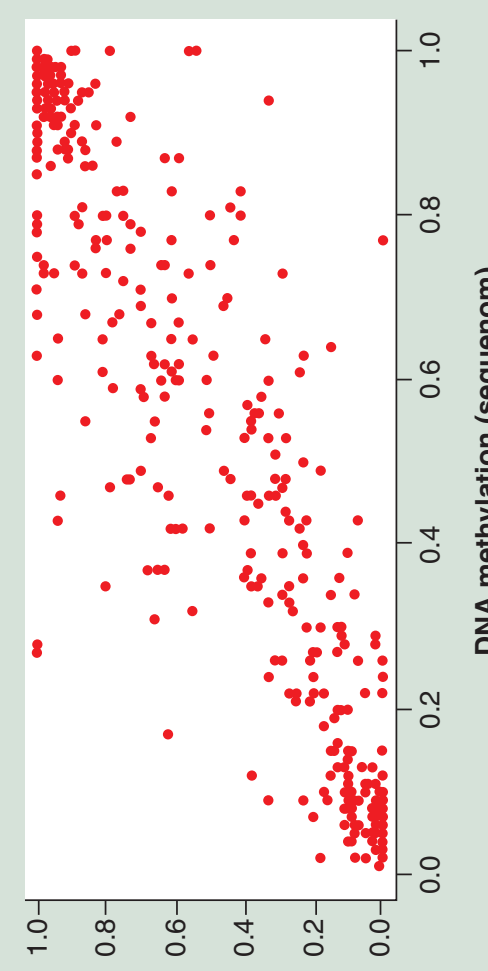

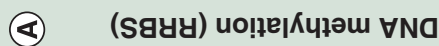

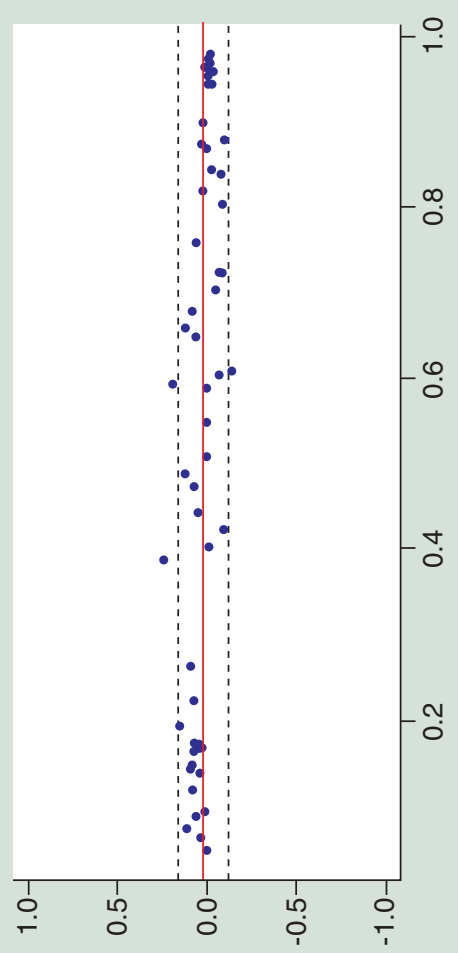

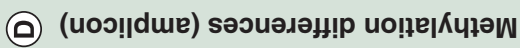

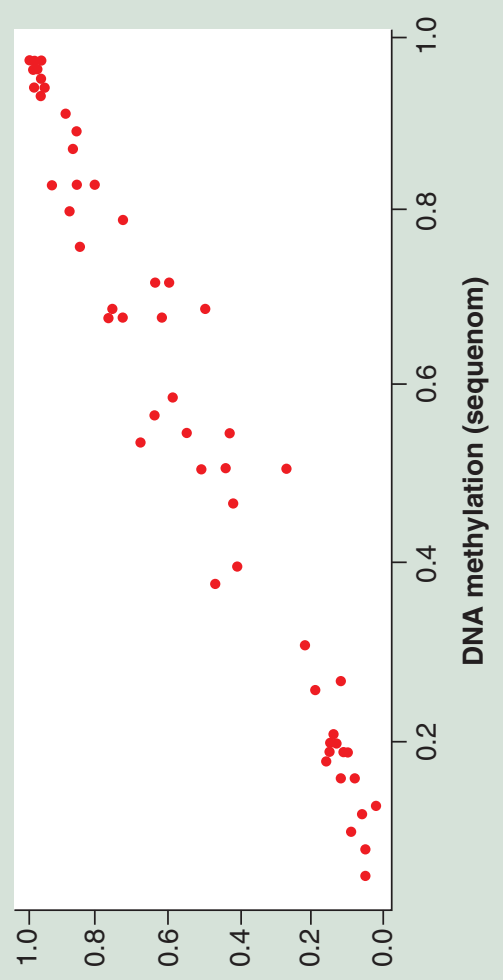

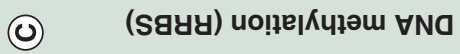

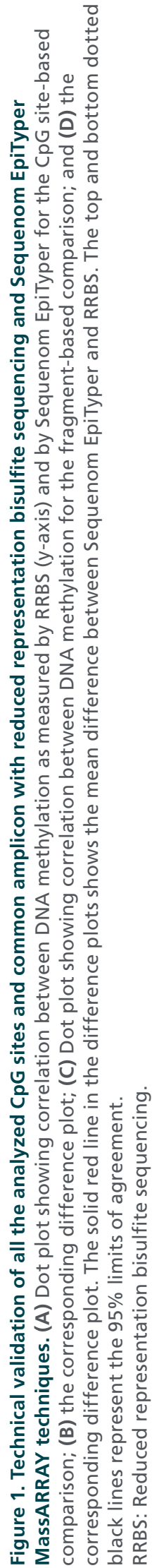




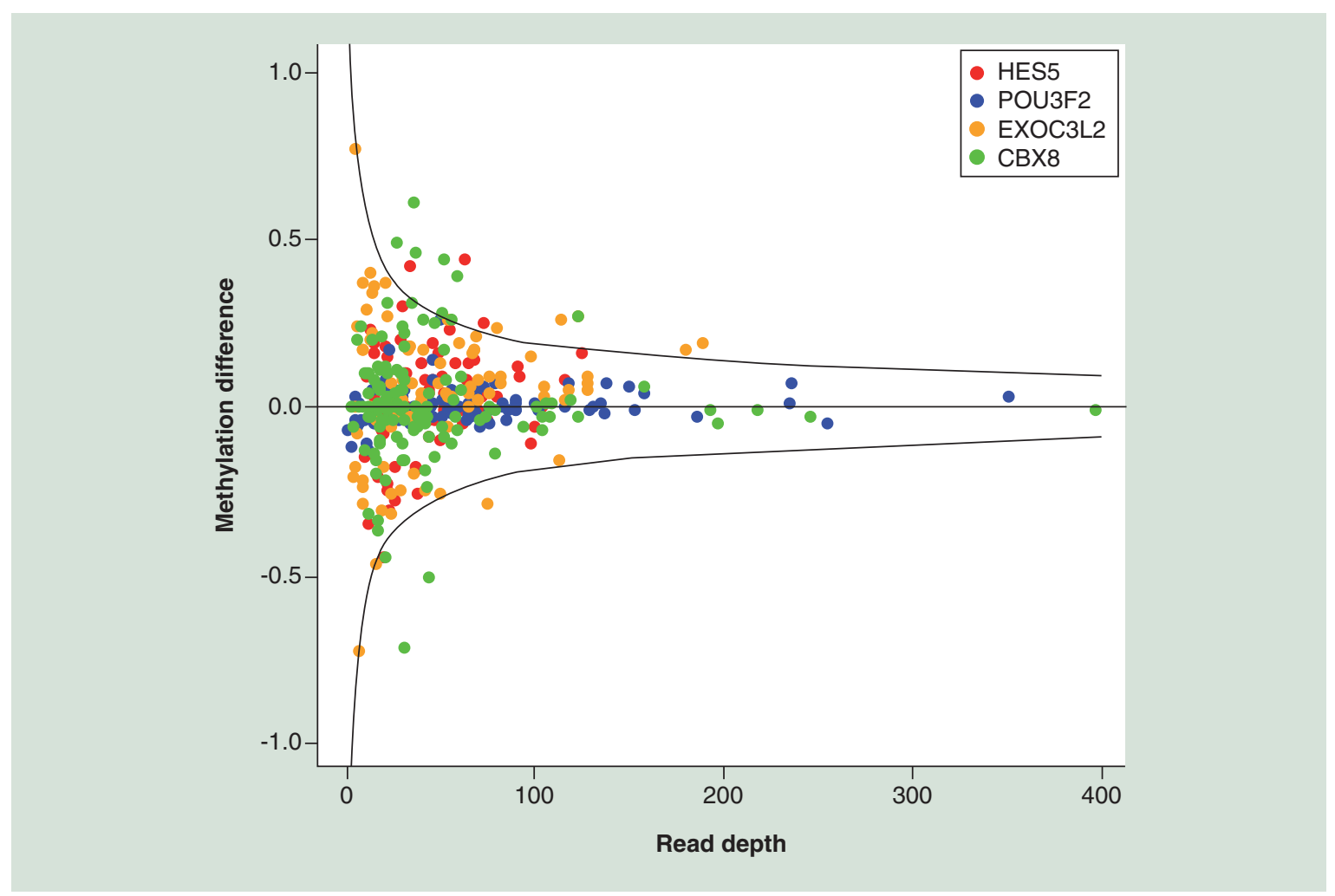

Figure 2. Relationship between methylation difference and reduced representation bisulfite sequencing read depth in analysed CpG sites. Y-axis represents the methylation difference between Sequenom EpiTyper platform and reduced representation bisulfite sequencing (in a scale of -1-1) and $\mathrm{x}$-axis shows the mapped read number of the corresponding CpG sites (combined data for four genes and each gene is highlighted with different color). The black lines indicate the $95 \%$ prediction interval at each read depth for the measured methylation difference, under the assumption that the measured difference is due to noise that scales as $1 /$ sqrt (read depth).

and 0.03 for HES5, EXOC3L2 and POU3F2, respectively. The average $\mathrm{CpG}$ methylation of these seven replicates also showed good concordance with RRBS $(\mathrm{r}=0.908$, $\mathrm{p}$-value $=0.00002$ and $\mathrm{SD}$ of bias $=0.107$, Supplementary Figure 6).

\section{Discussion}

Previous benchmark studies have compared the performance of several genome-wide methylation analysis platforms [11,12]. These analyses focused on genomewide correlation, detection of differentially methylated regions and the effect of sequencing depth. Here, we present the use of a targeted approach using the quantitative Sequenom EpiTyper assay to compare the methylation of four genes that were first analyzed by using a genome-wide approach (RRBS). We have used a panel of 15 cell lines that includes primary and metastatic melanoma as well as normal melanocyte cell lines for comparison. Further, we have performed multiple repeats in our Sequenom EpiTyper methylation analysis to provide a measure of technical variation in this process. In general, we see significant concordance between RRBS and Sequenom EpiTyper methylation patterns. Our observations are consistent with previous studies that demonstrated Sequenom EpiTyper platform to be a reliable targeted approach to validate genome-wide methylation data generated from RRBS [13-15].

By comparing RRBS and Sequenom EpiTyper methylation data, we demonstrate that methylation values between the two techniques are in better agreement if regions containing multiple $\mathrm{CpG}$ sites are compared instead of regions containing single CpG sites. There could be several reasons that might lead to increased variability when analyzing methylation data from single CpG sites. First, the technical platforms used to derive methylation calls (sequencing-based techniques and mass spectrometric-based techniques) have inherent differences in resolving methylation differences, which will affect the accuracy of methylation quantification. Second, a reduced number of reads covering a single site compared with a contig of sites can influence the methylation call. Finally, sampling-based variation could also contribute to the differences in methylation values between the platforms. 


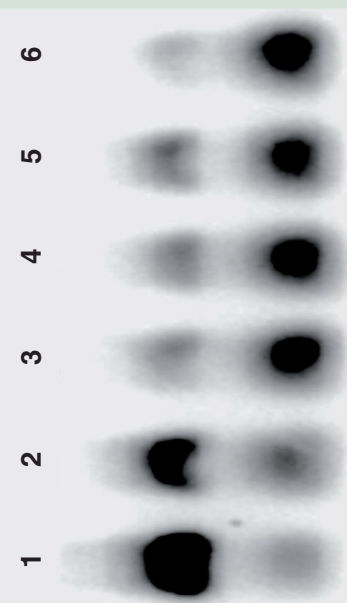

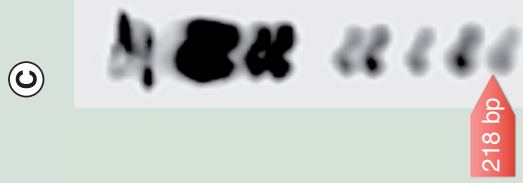

(๓)

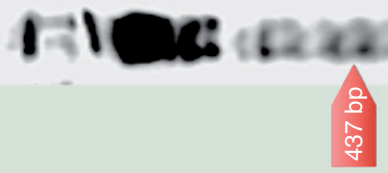

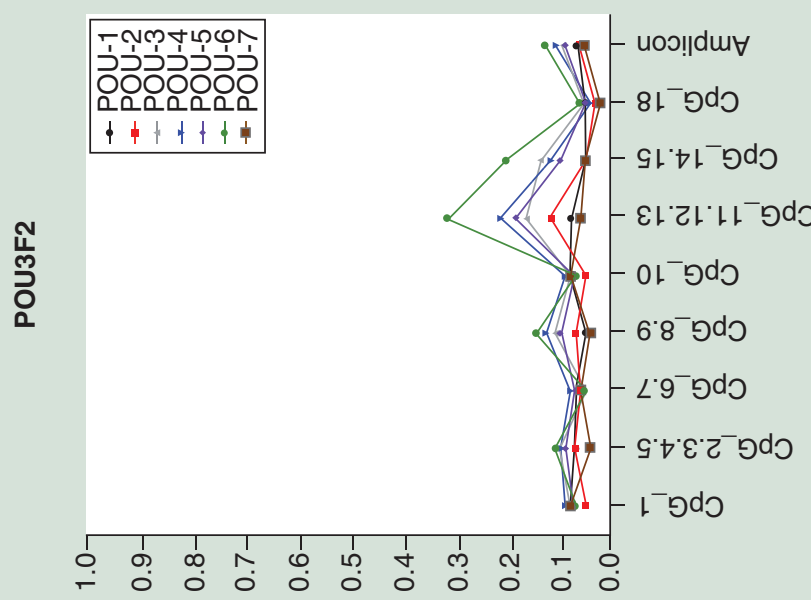

(1) ио!ฺејКчұәш $\forall$ Na
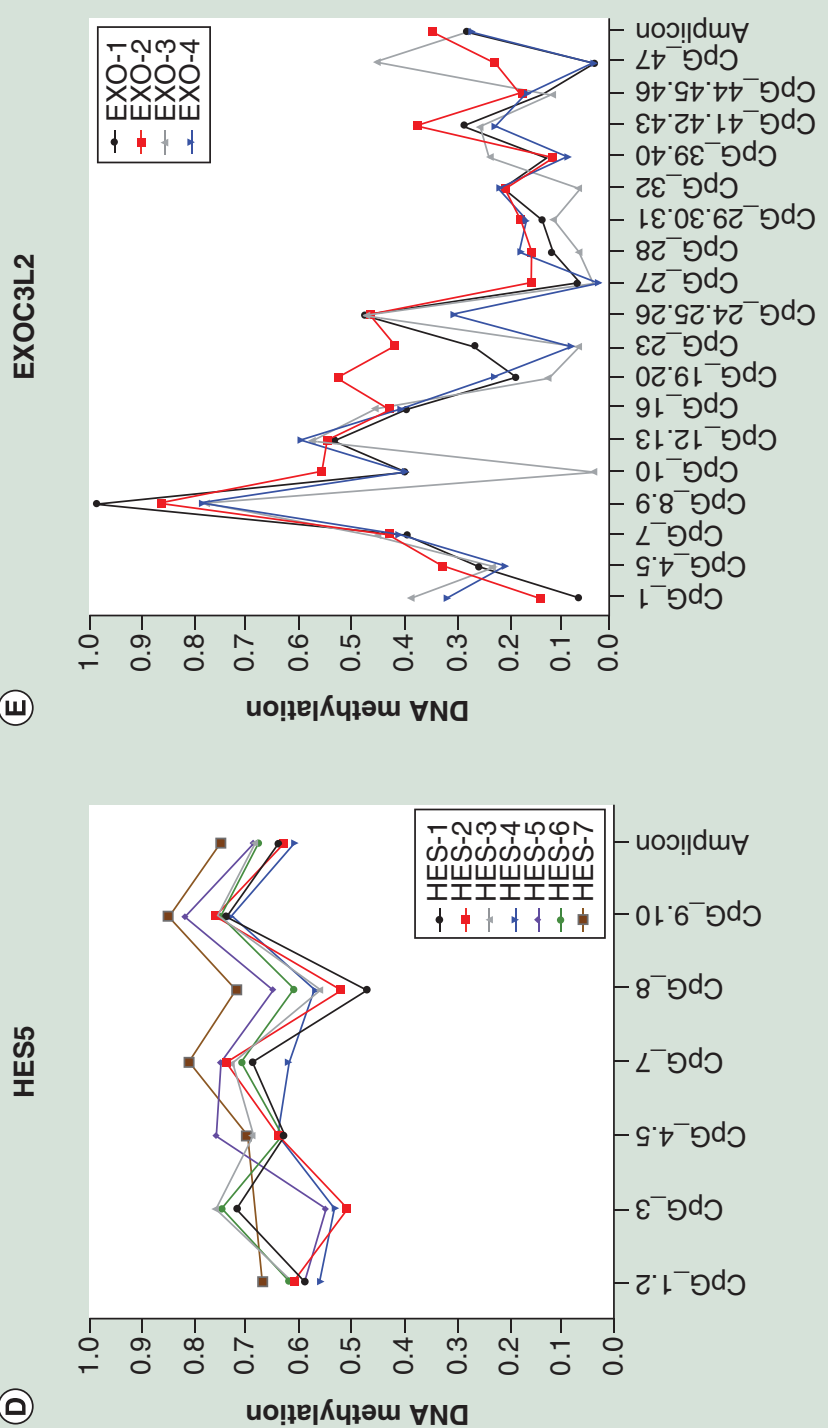
Figure 3. DNA methylation analysis of multiple technical replicates in Sequenom EpiTyper platform.

(A-C) Successful amplification of the technical replicates using EpiTyper primers for HES5, EXOC3L2 and POU3F2 (left to right). Ladder: 100 bp DNA ladder (Invitrogen). An additional replicate sample was prepared for each of these genes and is not included in this gel electrophoresis. (D-F) Methylation status of each CpG sites in the amplicon for replicate samples for HES5 (7 replicates), EXOC3L2 (4 replicates) and POU3F2 (7 replicates) (left to right). For HES5, EXOC3L2 and POU3F2, PCR gel of 6, 3 and 6 replicates is shown. Figure 3 shows examples of several adjacent CpG sites for which Sequenom EpiTyper was unable to resolve methylation values for individual sites (such as CpGs 4 and 5 and then 9 and 10 for the HES5 gene amplicon, see $x$-axis).

As expected, increased read depth in RRBS results in a better correlation with Sequenom EpiTyper methylation values. In fact, the improvement between RRBS and Sequenom EpiTyper methylation has a straightforward explanation: RRBS and Sequenom EpiTyper both give noisy measurements of the actual methylation, but, assuming independent reads, we expect the uncertainty in the RRBS measurement to have a component that scales as $1 / \sqrt{ }$ (read depth) (Figure 2). However, many CpG sites, in spite of the read depth being greater than ten, showed a large difference. It is possible that the mapping of reads results in erroneous methylation calls at some CpG sites. For mapping bisulfite sequenced reads, the mapping algorithms need to resolve $\mathrm{C}$ to $\mathrm{T}$ asymmetry [16] and can generate differences by mapping methylated reads more efficiently than unmethylated reads [17]. Therefore, inspection of read-mapping patterns might be an important step to consider before understanding sources of bias from RRBS experiments.

We detected notable variation in technical replicate samples in Sequenom EpiTyper assays for some CpG sites. This variability could be a result of the following: if adjacent $\mathrm{CpGs}$ cannot be resolved by Sequenom EpiTyper platform; if the CpGs under analysis are the first or last within an amplicon (these are more variable as previously reported by [4]); if the methylation value is intermediate (see Figure 4 and Supplementary Figure 7); and if suboptimal PCR performance biases the composition of the product under analysis. A previous study analyzed three genes in Sequenom EpiTyper platform and based on three PCR replicates they reported median standard errors of 0.02, 0.04 and 0.11 for these genes [4]. In our study, for the three analyzed genes we found the median standard error to be 0.007 (for POU3F2), 0.0205 (for HES5) and 0.0325 (for EXOC3L2) based on seven, seven and four replicates, respectively, see Supplementary Figure 7). These results are in agreement with previous reports of degree of variation in Sequenom EpiTyper platform. These results highlight the importance of including multiple replicates for validation to reduce sampling bias and PCR variation. An alternative strategy could be pooling multiple PCR replicates before profiling methylation patterns to reduce sampling noise. However, this needs to be tested in future studies.
DNA methylation at adjacent CpG sites is correlated $[11,12]$. Further, neighboring $\mathrm{CpGs}$ are likely to have similar experimental properties (such as similar read depth and similar PCR characteristics). Although methylation levels are generally similar in adjacent sites, there are exceptions. In our analysis, we observe substantial variation in specific CpG sites compared with their adjacent sites. For example, EXOC3L2 CpGs 8,9 (aggregated sites, see Figure 3E), HES5 CpG8 and POU3F2 CpGs 11,12,13 (aggregated sites, see Figure 3F). Previously, we have demonstrated substantial methylation variation in adjacent $\mathrm{CpG}$ sites within single fragments in a relatively homogeneous cell population of neutrophils [18]. Mechanistic studies on adjacent DNA methylation dynamics are lacking and therefore firm conclusions cannot be drawn about the biological implications of adjacent $\mathrm{CpG}$ site methylation. However, it is plausible that some specific $\mathrm{CpG}$ sites in a contig might be under selective transcriptional control and therefore are necessary to alter binding of transcription factors, insulators or enhancer elements that are required to regulate transcription $[19,20]$.

Although we analyzed sequencing-based RRBS data, the findings from this study could be utilized in other platforms for selection of $\mathrm{CpG}$ sites for validation. For example, Illumina's high density methylation array is currently the most widely used platform for profiling large number of samples due to the relative ease of extracting methylation calls. Almost half of

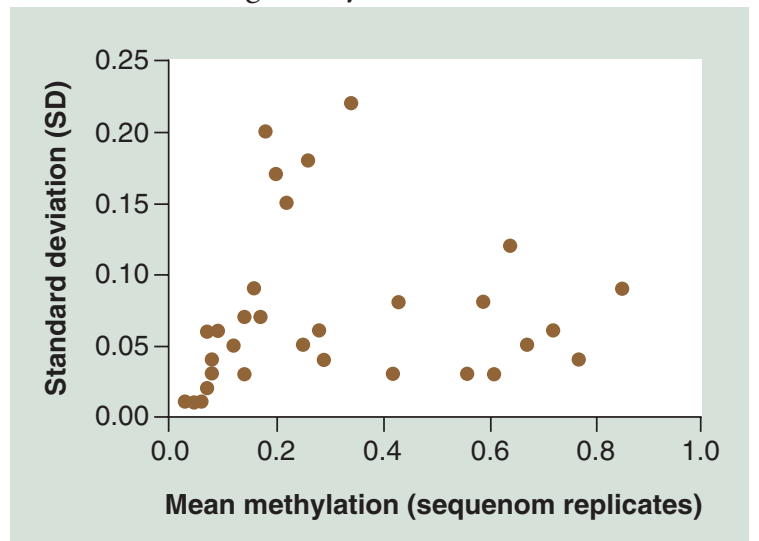

Figure 4. Relationship of standard deviation and methylation in Sequenom EpiTyper replicates.

$\mathrm{X}$-axis: mean methylation of all the replicates in $\mathrm{CpG}$ sites; y-axis: corresponding standard deviation (SD). 
the probes on $450 \mathrm{~K} \mathrm{CpG}$ array were located around promoters and often one promoter contains multiple probes (average number of $\mathrm{CpG}$ probe/protein coding promoter is 9) [21]. The results from this work reveal potential sources of bias that can be used to refine experimental design for studies that are focused on validating methylation data generated from large-scale DNA methylation studies.

\section{Materials \& methods}

\section{Cell line experiments}

In total, 15 cell lines were used in this article. These cell lines were obtained from America Type Culture Collection (VA, USA). These cell lines are: WM-115 (ATCC ${ }^{\circledR}$ CRL-1675'M), WM266-4 (ATCC CRL$1676^{\mathrm{TM}}$ ), Hs688(A).T (ATCC CRL-7425 ${ }^{\mathrm{TM}}$ ) and Hs688(B).T ATCC CRL-7426 ${ }^{\mathrm{TM}}$ ). These cell lines were grown and maintained in Minimum Essential Media and supplemented with $1 \%$ penicillin-streptomycin (Gibco, NY, USA) and 10\% fetal bovine serum (Moregate biotech, Australia \& New Zealand) under standard conditions $\left(5 \% \mathrm{CO}_{2}, 37^{\circ} \mathrm{C}\right.$, humidified atmosphere). Three normal melanocyte cell lines (Hemn-LP, HemA-LP and Mel-ST) were obtained from Robert Weinberg, MIT, USA. Eight additional cell lines were used for preparing the RRBS libraries. For these cell lines (WM793, WM853-2, WM278, WM39, WM1341D, and WM15552C, WM75, WM373), DNA material was obtained from The Wistar Institute (PA, USA).

\section{RRBS library preparation \& sequencing}

RRBS libraries were prepared according to previously published protocols [22]. Briefly, genomic DNA was digested with the MspI enzyme followed by end-repair and ligation of sequencing adaptors. The fragments were then size-selected ( $40-220 \mathrm{bp})$ and bisulfite converted prior to a PCR amplification step. The quality of the libraries was checked using a bioanalyzer, and four libraries (per flow cell lane) were sequenced on an Illumina HiSeq2500 machine (100 bp, single-ended run).

\section{RRBS data analysis}

Processing of the reduced representation bisulfite sequencing data was analyzed using our in-house bioinformatics pipeline [23]. Analysis of mapped RRBS data and extraction of $\mathrm{CpG}$ methylation was performed using DMAP (differential methylation analysis package) tools [24].

Sequenom EpiTyper DNA methylation assay

The primers used for analyzing four genes described here were designed using the EpiTyper assay designer [25]. The sequences of the primers are given in Supplementary Table 2. Briefly, $1 \mu \mathrm{g}$ of genomic DNA was bisulfite converted using the EZ DNA methylation kit (Zymo Research) using the 'alternative cycle protocol' as stated by the manufacturer. PCR was performed with the following cycling protocol: $15 \mathrm{~min}$ at $94^{\circ} \mathrm{C}, 45$ cycles of $\left(20 \mathrm{~s}\right.$ at $94^{\circ} \mathrm{C}, 30 \mathrm{~s}$ at optimal annealing temperature, $1 \mathrm{~min}$ at $72^{\circ} \mathrm{C}$ ), $3 \mathrm{~min}$ at $72^{\circ} \mathrm{C}$ for extension and hold at $4^{\circ} \mathrm{C}$. The mass spectrometrybased Sequenom EpiTyper assay was performed using the manufacturers' protocol on one 384-well plate (Sequenom Facility, University of Auckland). During the mass spectrometry run, the Sequenom EpiTyper assay generates spectrum quality data for each $\mathrm{CpG}$ site that were analyzed [26]. Our spectrum quality data were of acceptable quality to be used for the subsequent analysis. The spectrum characteristics of the assay were analyzed at the facility and the methylation values for each $\mathrm{CpG}$ site in the designed amplicons were obtained. Analysis of methylation data was performed using excel and R statistical software (Version 3.02).

\section{Code availability}

Standard R commands were used to perform statistical tests and generate the figures. The codes are available from the authors on request (aniruddha.chatterjee@ otago.ac.nz). In addition, data analysis of single genes was performed using Prism Graphpad software.

\section{Supplementary data}

To view the supplementary data that accompany this paper please visit the journal website at: www.futuremedicine.com/ doi/full/10.2217/epi-2016-0176

\section{Author contributions}

A Chatterjee, E Macaulay, MR Eccles and IM Morison conceptually designed the project. A Chatterjee analyzed the data and wrote the first draft of the manuscript together with $E$ Macaulay. A Ahn performed the EpiTyper PCR experiments. $J L$ Ludgate prepared multiple RRBS libraries. PA Stockwell developed the computational pipeline for data analysis and performed alignments and helped in data analysis. RJ Weeks helped in the design of the EpiTyper assays and interpretation of data. MF Parry contributed to the statistical analysis and integration of the data. IM Knarston and TJ Foster contributed to the Sequenom EpiTyper PCR experiments and data analysis, respectively. All authors read, contributed and approved the final manuscript.

\section{Acknowledgements}

We gratefully acknowledge the New Zealand Institute for Cancer Research Trust and University of Otago Leading Thinkers Advancement Campaign for supporting A Chatterjee and MR 
Eccles respectively. We thank Anna Leichter and Euan Rodger for helping with figure preparation. We gratefully acknowledge the support of the Sequenom facility and in particular help from Phillip Shepherd (Liggins Institute, Auckland) for the Sequenom EpiTyper DNA methylation assay.

\section{Financial \& competing interests disclosure}

We acknowledge the financial support provided by the Maurice Wilkins Centre for Molecular Biodiscovery, Health Research South of the Dunedin School of Medicine, and Maurice Phyllis Paykel Trust and the Healthcare Charitable Otago Trust. The authors have no other relevant affiliations or financial involvement with any organization or entity with a financial interest in or financial conflict with the subject matter or ma- terials discussed in the manuscript apart from those disclosed. No writing assistance was utilized in the production of this manuscript.

\section{Ethical conduct of research}

The experiments and analysis described here were carried out in accordance with the Health and Disability Ethics Committee of New Zealand protocol LRS/11/02/002/AM04.

\section{Open access}

This work is licensed under the Attribution-NonCommercial-NoDerivatives 4.0 Unported License. To view a copy of this license, visit http://creativecommons.org/licenses/bync-nd/4.0/

\section{Summary points}

- Validating this next generation sequencing (NGS)-based DNA methylation data is an important step for meaningful translation of findings. However, there has been limited assessment of different platforms to validate methylation data from NGS.

- We provide a comparative assessment of DNA methylation patterns between a NGS-based, genome-wide platform (reduced representation bisulfite sequencing) with a targeted, mass spectrometry based platform (Sequenom EpiTyper). We compared the DNA methylation patterns of four genes in 15 cell lines (covering 52 CpG sites).

- We show that the accuracy of validation of DNA methylation profiles between the two methods substantially improves if results from multiple and adjacent CpG sites are combined (i.e., investigating a region) rather than at single $\mathrm{CpG}$ sites.

- Increased read number improves accuracy of reduced representation bisulfite sequencing results and we provide estimates of variation at different read number cut offs.

- By using series of replicates, we demonstrate variation within technical replicate samples analyzed by Sequenom EpiTyper, which indicates that the use of replicate samples will enhance precision.

- This work reveals potential sources of bias that can be used to refine experimental design for studies that are focused on validating methylation data generated from large-scale DNA methylation studies.

\section{References}

Papers of special note have been highlighted as: $\bullet$ of interest;

-• of considerable interest

1 Meissner A, Gnirke A, Bell GW, Ramsahoye B, Lander ES, Jaenisch R. Reduced representation bisulfite sequencing for comparative high-resolution DNA methylation analysis. Nucleic Acids Res. 33(18), 5868-5877 (2005).

2 Chatterjee A, Rodger EJ, Morison IM, Eccles MR, Stockwell PA. Tools and strategies for analysis of genome-wide and gene-specific dna methylation patterns. In: Oral Biology. Seymour GJ, Cullinan MP, Heng NCK (Eds). Springer, New York, NY, USA, 249-277 (2017).

3 Olkhov-Mitsel E, Bapat B. Strategies for discovery and validation of methylated and hydroxymethylated DNA biomarkers. Cancer Med. 1(2), 237-260 (2012).

4 Coolen MW, Statham AL, Gardiner-Garden M, Clark SJ. Genomic profiling of $\mathrm{CpG}$ methylation and allelic specificity using quantitative high-throughput mass spectrometry: critical evaluation and improvements. Nucleic Acids Res. 35(18), e119 (2007).

- Described the principle and potential biases of Sequenom EpiTyper platform for methylation analysis.
5 Christensen BC, Kelsey KT, Zheng S et al. Breast cancer DNA methylation profiles are associated with tumor size and alcohol and folate intake. PLoS Genet. 6(7), e1001043 (2010).

6 Breitling LP, Yang R, Korn B, Burwinkel B, Brenner H. Tobacco-smoking-related differential DNA methylation: $27 \mathrm{~K}$ discovery and replication. Am. J. Hum. Genet. 88(4), 450-457 (2011).

7 Figueroa ME, Lugthart S, Li Y et al. DNA methylation signatures identify biologically distinct subtypes in acute myeloid leukemia. Cancer Cell 17(1), 13-27 (2010).

8 Farthing CR, Ficz G, Ng RK et al. Global mapping of DNA methylation in mouse promoters reveals epigenetic reprogramming of pluripotency genes. PLoS Genet. 4(6), e1000116 (2008).

9 Liang P, Song F, Ghosh S et al. Genome-wide survey reveals dynamic widespread tissue-specific changes in DNA methylation during development. BMC Genomics 12(1), 231 (2011).

10 Chatterjee A, Stockwell PA, Ahn A, Rodger EJ, Leichter AL, Eccles MR. Genome-wide methylation sequencing of paired primary and metastatic cell lines identifies common DNA methylation changes and a role for EBF3 
as a candidate epigenetic driver of melanoma metastasis. Oncotarget 8(4), 6085-6101 (2016).

-. Described the first framework for identifying epigenetic driver events in cancer metastasis.

11 Bock C, Tomazou EM, Brinkman AB et al. Quantitative comparison of genome-wide DNA methylation mapping technologies. Nat. Biotechnol. 28(10), 1106-1114 (2010).

- Benchmarking paper that compared reduced representation bisulfite sequencing, MethylCap-seq and MeDIP-seq and one array-based platform: $27 \mathrm{~K}$ bead array for methylation analysis.

12 Harris RA, Wang T, Coarfa C et al. Comparison of sequencing-based methods to profile DNA methylation and identification of monoallelic epigenetic modifications. Nat. Biotechnol. 28(10), 1097-1105 (2010).

-• Benchmarking paper that compared MethylC-seq, reduced representation bisulfite sequencing, MeDIP-seq and MBDseq for methylation analysis.

13 Jin S, Lee YK, Lim YC et al. Global DNA hypermethylation in down syndrome placenta. PLoS Genet. 9(6), e1003515 (2013).

14 Akalin A, Garrett-Bakelman FE, Kormaksson M et al. Base-pair resolution DNA methylation sequencing reveals profoundly divergent epigenetic landscapes in acute myeloid leukemia. PLoS Genet. 8(6), e1002781 (2012).

15 Couldrey C, Brauning R, Bracegirdle J, MaClean P, Henderson HV, Mcewan JC. Genome-wide DNA methylation patterns and transcription analysis in sheep muscle. PLoS ONE 9(7), e101853 (2014).

16 Xi Y, Li W. BSMAP: whole genome bisulfite sequence MAPping program. BMC Bioinformatics 10, 232 (2009).

17 Chatterjee A, Rodger EJ, Stockwell PA, Weeks RJ, Morison IM. Technical considerations for reduced representation bisulfite sequencing with multiplexed libraries. J. Biomed. Biotechnol. 2012, 741542 (2012).
18 Chatterjee A, Stockwell PA, Rodger EJ et al. Genomewide DNA methylation map of human neutrophils reveals widespread inter-individual epigenetic variation. Sci. Rep. 5 , 17328 (2015).

-• Described extensive interindividual DNA methylation variation in human neutrophils.

19 Zhu WG, Srinivasan K, Dai Z et al. Methylation of adjacent CpG sites affects Sp1/Sp3 binding and activity in the p21(Cip1) promoter. Mol. Cell. Biol. 23(12), 4056-4065 (2003).

20 Wang H, Maurano MT, Qu H et al. Widespread plasticity in CTCF occupancy linked to DNA methylation. Genome Res. 22(9), 1680-1688 (2012).

21 Bibikova M, Barnes B, Tsan C et al. High density DNA methylation array with single $\mathrm{CpG}$ site resolution. Genomics 98(4), 288-295 (2011).

22 Chatterjee A, Rodger EJ, Stockwell PA, Le Mee G, Morison IM. Generating multiple base-resolution DNA methylomes using reduced representation bisulfite sequencing. Methods Mol. Biol. 1537, 279-298 (2017).

23 Chatterjee A, Stockwell PA, Rodger EJ, Morison IM. Genome-scale DNA methylome and transcriptome profiling of human neutrophils. Sci. Data 3, 160019 (2016).

24 Stockwell PA, Chatterjee A, Rodger EJ, Morison IM. DMAP: differential methylation analysis package for RRBS and WGBS data. Bioinformatics 30(13), 1814-1822 (2014).

-. Described the DMAP tool which contains suite of statistical and analytical tool for methylation analysis.

25 Sequenom. EpiDesigner BETA. http://www.epidesigner.com/

26 Ehrich M, Zoll S, Sur S, Van Den Boom D. A new method for accurate assessment of DNA quality after bisulfite treatment. Nucleic Acids Res. 35(5), e29 (2007). 